\title{
Adaptive Laser Compensation for Aero-Optics and Atmospheric Disturbances
}

\author{
Matthew R. Whiteley* \\ MZA Associates Corporation, Dayton, OH 45459-2678, U.S.A. \\ J. S. Gibson ${ }^{\dagger}$ \\ University of California, Los Angeles, CA 900095-1597, U.S.A.
}

\begin{abstract}
Performance of adaptive optics (AO) compensation for beam control is quantified via wave-optical propagation, sensing, and control methods using wind tunnel measurements ${ }^{1}$ of aero-optical disturbances in addition to Kolmogorov turbulence distributed over a laser path. For Kolmogorov turbulence the residual phase variance scales as $\left(f_{G} / f_{3 d B}\right)^{5 / 3}$, where $f_{G}$ is the Greenwood frequency for the propagation path ${ }^{2}$ and $f_{3 d B}$ is the error-rejection bandwidth of the classical AO control. It is shown that the residual phase variance with classical AO control, when normalized to the open-loop, scales as $\left(f_{A} / f_{3 d B}\right)^{\gamma}$, where $\gamma$ is an arbitrary power and $f_{A}$ is a characteristic frequency of the aero-optical disturbance determined from a linear fit of the compensation data with increasing bandwidth. AO system latency degrades performance, especially with high-bandwidth control. When operating at a fixed but modest sampling frequency with appreciable latency, $\mathrm{AO}$ compensation performance can be significantly enhanced by application of an adaptive control augmentation based on lattice filtering of the residual wavefront sensor gradients, as was implemented in the wave-optics simulations. A classical controller operating at $200 \mathrm{~Hz}$ bandwidth with $>400 \mu$ sec latency has a limited ability to compensate the aero-optical and free-stream disturbances. By application of the adaptive feed-forward control, laser peak irradiance is shown to increase by a factor of $\mathbf{2 . 5}$ or more compared to classical AO feedback control.
\end{abstract}

\section{Nomenclature}

$\beta \quad$ Gain of classical adaptive optics control loop

$\Delta t \quad$ Latency of an adaptive optics compensation system (s)

Compensation scaling power for aero-optical disturbances $(\mathrm{Hz})$

$\lambda \quad$ Wavelength of laser $(\mathrm{m})$

Optical path difference for aero-optics disturbance $(\mathrm{m})$

Mass density of air $\left(\mathrm{kg} / \mathrm{m}^{3}\right)$

Fried's coherence diameter for free-stream Kolmogorov turbulence (m)

Variance of aero-optical wavefront disturbance $\left(\mathrm{rad}^{2}\right)$

Residual variance of aero-optical wavefront disturbance with compensation $\left(\mathrm{rad}^{2}\right)$

Diameter of laser transmitting aperture $(\mathrm{m})$

$D_{t} \quad$ Diameter of laser transmitter aircraft turret $(\mathrm{m})$

$f \quad$ Temporal frequency $(\mathrm{Hz})$

$f_{3 \mathrm{~dB}}$ Adaptive optics error rejection bandwidth $(\mathrm{Hz})$

$f_{A} \quad$ Compensation scaling frequency for aero-optical disturbances $(\mathrm{Hz})$

$f_{G} \quad$ Greenwood frequency for free-stream Kolmogorov turbulence $(\mathrm{Hz})$

$f_{s} \quad$ Sampling frequency of an adaptive optics control sensor $(\mathrm{Hz})$

$k_{1} \quad$ Gain of adaptive control loop augmentation

*Vice President, Senior Scientist, 6651 Centerville Business Parkway Suite B, Member AIAA.

${ }^{\dagger}$ Professor, Mechanical and Aerospace Engineering Department, 420 Westwood Plaza \#38-137, Box 951597. 
$S_{h} \quad$ Strehl ratio degradation associated with wavefront aberrations other than tilt (higher-order)

\section{Introduction}

ERODYnamic disturbances are well known to produce optical degradations when an aircraft turret is used A as a laser projection platform..$^{3,4}$ The most devastating of these are when the laser is directed into the aft quadrant, where the beam must propagate through separated flow. ${ }^{5}$ Phase compensation by adaptive optics (AO) has proven to be a valuable technology for overcoming the degrading effects of atmospheric turbulence in optical systems for imaging and beam projection. ${ }^{6,7}$ It is natural that AO be considered for use in compensating aero-optical disturbances, as the effects are in principal measurable given sufficient reference signals and adequate sensors. However, there is little information available for determining the suitability of an AO design for use in compensating aero-optical degradations.

We have made use of the latest available measurements of aero-optical disturbances to quantify the utility of AO technology for laser compensation. To this end, we have employed wave-optics simulations of a candidate AO system. Assessment of AO compensation for aero-optics reveals an important fact. While AO can improve propagation performance above the level with no compensation, classical control methods limit the substantial benefit to be gained from AO. Performance degrades as the severity of disturbance conditions increases. In recent years, adaptive control methods have been developed for higher-order wavefront correction in adaptive optics. ${ }^{8}$ These techniques have been shown to substantially improve AO compensation for laser beam projection systems. ${ }^{9}$ It is therefore natural to consider application of this technology to the problem of adaptive optics for aircraft laser systems suffering appreciable aero-optics disturbances in addition to those imparted by free-stream turbulence. Evaluation of adaptive control for AO compensation with aero-optics and free-stream turbulence is the primary subject of this study.

The remainder of this paper is structured as follows. In Sec. II we discuss the aero-optical disturbances and wave-optics models employed in our AO compensation simulations. Through analysis of AO compensation performance, we establish limitations with classical AO control resulting from finite bandwidth and net AO latency in Sec. III. Adaptive control methods are outlined in Sec. IV. The advantage of AO performance with adaptive control to performance with classical control is quantified. Results are shown for compensation of aero-optics disturbances only, free-stream turbulence only, and combined aero-optics and free-stream turbulence. We draw our conclusions in Sec. V.

\section{Disturbance modeling and adaptive optics simulation}

Wind-tunnel data collected at the United States Air Force Academy (USAFA) was used to quantify aerooptical disturbances under variable aerodynamic conditions. In these tests a 12" $(30 \mathrm{~cm})$ hemisphere was mounted to a cylinder to represent a typical turret configuration on an aircraft for use as a laser projection platform. The turret was equipped with a an aperture through which optical aberrations could be measured. The turret model was affixed to the side of the wind tunnel and a suite of optical characterizations were made over the aperture during wind tunnel flow operation at Mach 0.4. ${ }^{1}$ A subset of the optical measurements included a high-speed 8x8 subaperture Shack-Hartmann wavefront sensor (WFS) operating at $78.125 \mathrm{kHz}$ sampling frequency. The wavefront sensor data was reconstructed to phase maps over the 3" measurement aperture to quantify the aero-optical disturbance. The reconstructed wavefront data was normalized so that to derive the proper optical path difference (OPD) $\phi^{\prime}$ for the desired conditions, the WFS reconstruction $\phi$ was scaled according to: ${ }^{5}$

$$
\phi^{\prime}=\left(M^{\prime^{2}} D_{t}^{\prime} \frac{\rho^{\prime}}{\rho_{h=0}}\right) \phi,
$$

where $M^{\prime}$ is the desired Mach number for the turret platform, $D_{t}^{\prime}$ is the diameter of the platform turret, $\rho^{\prime}$ is the density of air for the desired altitude of operation, and $\rho_{h=0}$ is the air density at sea level (1.225 $\mathrm{kg} / \mathrm{m}^{3}$ for standard atmosphere). In our simulation-based studies we desire to represent aero-optical effects for a turret diameter of 50 inches $\left(D_{t}=127 \mathrm{~cm}\right)$ for an aircraft with speed $M^{\prime}=0.3$. The air density was assumed to be $\rho^{\prime}=0.918 \mathrm{~kg} / \mathrm{m}^{3}$, consistent with a flight altitude near $10 \mathrm{kft}$ above sea level. Under these scaling conditions, the wind tunnel data for $130^{\circ}$ turret angle have a peak-to-valley OPD of $\sim 3 \lambda(0.19 \lambda$ rms) for $\lambda=1 \mu \mathrm{m}$ wavelength.

In addition to scaling the magnitude of the wavefront for an assumed turret diameter, aircraft speed, and 
air density, the temporal evolution of the disturbance must also be scaled to for the desired geometry. To affect the proper temporal scaling when changing the platform speed or turret diameter, one needs to keep the Strouhal number $\left(f D_{t} / M\right)$ constant, where $f$ is the temporal frequency of the data. It follows from this assumption that the scaled frequency will be computed as:

$$
f^{\prime}=f\left(\frac{M^{\prime}}{M}\right)\left(\frac{D_{t}}{D_{t}^{\prime}}\right)
$$

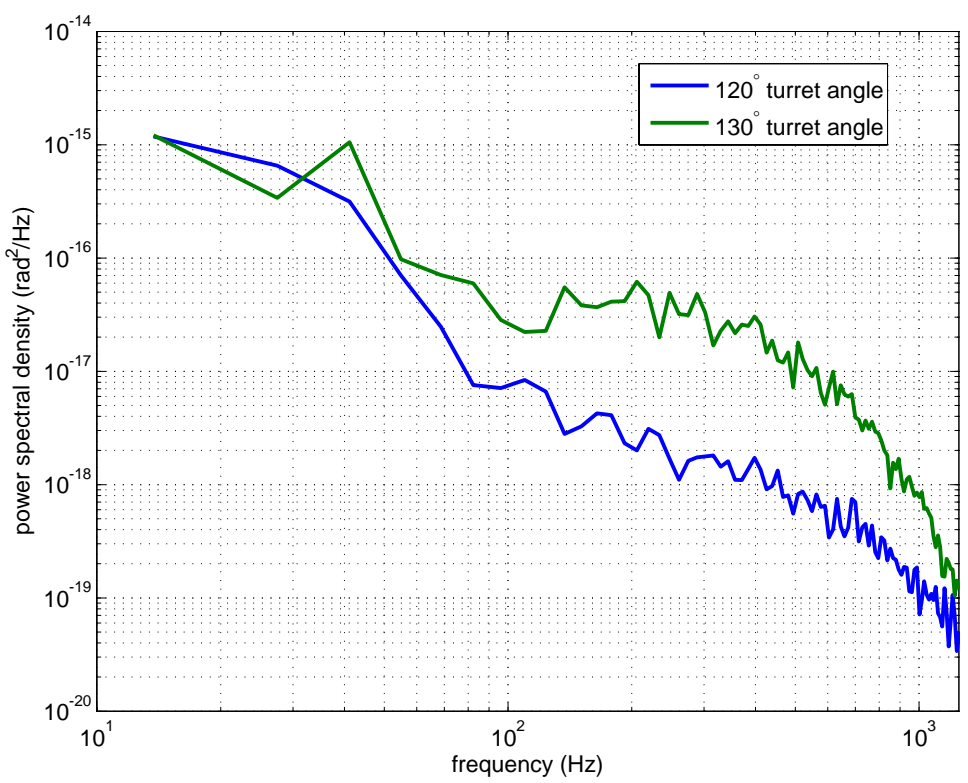

Figure 1. Temporal power spectral density for scaled wind tunnel aerooptics disturbances with $120^{\circ}$ and $130^{\circ}$ turret angle. Enhancement of high-frequency disturbance at $130^{\circ}$ is attributed to the shear-layer.

To test closed-loop adaptive optics compensation against various representations of aerooptical disturbances, we set up the simple WaveTrain ${ }^{\mathrm{TM} 10}$ compensation simulation shown in Fig. 2. This wave-optics system includes a point reference that propagates through an atmospheric path (may contain free-stream turbulence phase screens) in addition to an aero-optical path to provide light to sensors on the laser projection platform. This laser platform includes closed-loop steering mirror and focal-plane tracking system as well as a closed-loop deformable mirror (DM) and Hartmann WFS adaptive optics system. The AO system is modeled with 16 subapertures over the $28 \mathrm{~cm}$ full aperture diameter $(1.75 \mathrm{~cm}$ subapertures) and no central obscuration. The DM and WFS are set up with a Fried geometry, implying that the actuators are
Given the desired configuration and these scaling relations, the original data (after being down-sampled by a factor of 4) was scaled down to an update rate of $3.5156 \mathrm{kHz}$ for input to the wave-optics simulations. Fig. 1 shows the temporal power spectral density (PSD) of the scaled aero-optics wind tunnel data for $120^{\circ}$ and $130^{\circ}$ turret angle. The most notable difference in these PSDs is the additional high-frequency component at $130^{\circ}$ turret angle for $f>100 \mathrm{~Hz}$. This highfrequency component has been associated with an aero-optical shear-layer in previous studies. ${ }^{5}$ Thus, high-frequency $(f>$ $144 \mathrm{~Hz}$ ) filtered versions of the original temporal sequences are considered separately in our simulations to isolate the impact of the shear layer. For $130^{\circ}$ turret angle the high-frequency shear layer was observed to have a peak-to-valley OPD of $\sim 1 \lambda(0.10 \lambda \mathrm{rms}, \lambda=1 \mu \mathrm{m}$ wavelength $)$ thus constituting an appreciable component of the total disturbance.

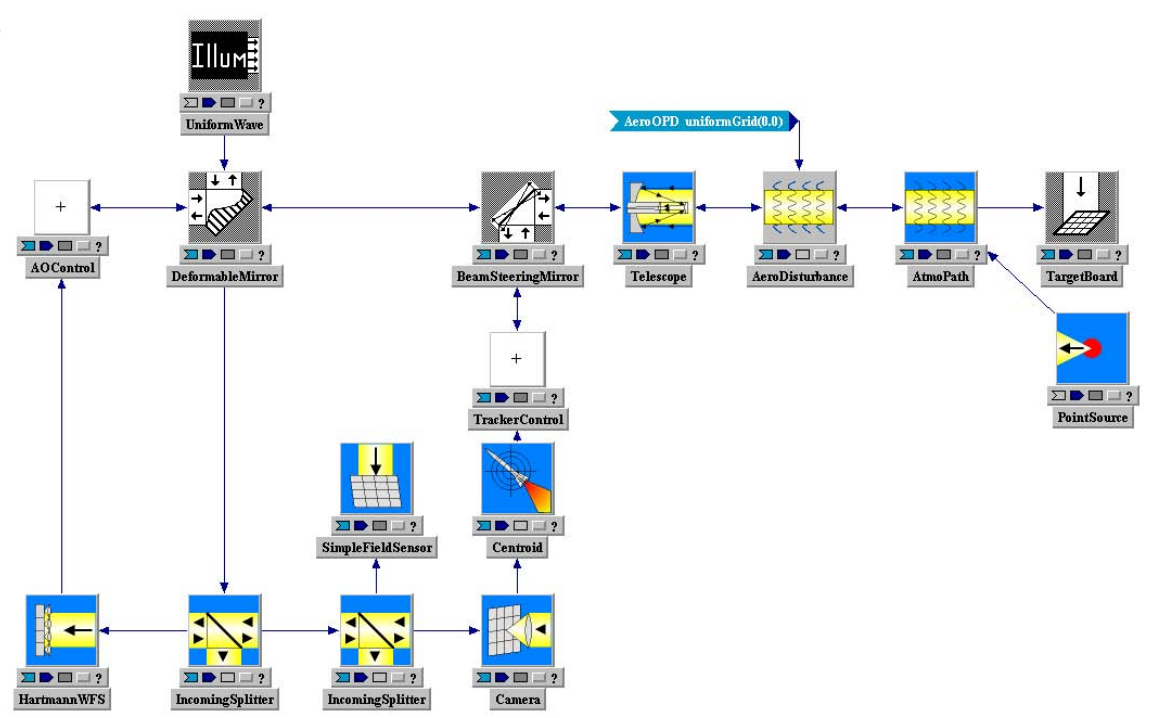

Figure 2. WaveTrain ${ }^{\mathrm{TM}}$ system diagram for the wave-optics propagation simulation used for adaptive optics compensation of aero-optical disturbances. 
spaced at $1.75 \mathrm{~cm}$ at the corners of each subaperture. As the simulation is executed in time, the aero-optical disturbance may be updated given any appropriate modeling data supplied to the "AeroOPD" input.

\section{Limits of classical adaptive optics control}

\section{III.A. Bandwidth limitations and compensation scaling frequencies}

The AO simulation implements a classical discrete integrator control law where the DM actuator commands $c$ at time $t_{k+1}$ are given by

$$
c\left(t_{k+1}\right)=c\left(t_{k}\right)+\beta \epsilon\left(t_{k}\right),
$$

where $\beta$ is the loop gain applied to the reconstructed wavefront error $\epsilon\left(t_{k}\right)$ at each time step $t_{k}$. If the control system has no latency and the response time of the mirror is instantaneous, then the error rejection function for the controller is modeled as

$$
E R J(f)=\left[1+\left(\frac{f}{f_{3 d B}}\right)^{-2}\right]^{-1},
$$

where the error rejection bandwidth $f_{3 \mathrm{~dB}}$ is commonly ${ }^{11,12}$ defined as

$$
f_{3 \mathrm{~dB}} \equiv \frac{\beta f_{s}}{2 \pi},
$$

and $f_{s}$ designates the sampling frequency of the discrete-time system.

The high-speed wind tunnel wavefront reconstructions were scaled in magnitude and frequency and employed in our adaptive optics simulation model to represent aero-optical disturbances. AO performance was quantified in terms of a compensated tilt-removed Strehl ratio for $0 \mathrm{~Hz} \leq f_{3 \mathrm{~dB}} \leq 500 \mathrm{~Hz}$. The orderly progression of Strehl ratio with increasing bandwidth suggested that AO compensation of these aero-optical disturbances follow an appropriate analytic form. To analyze AO compensation for the scaled wind tunnel disturbances, and by analogy with the analysis of bandwidth specifications for compensation of free stream turbulence, ${ }^{2}$ we tested the simulation results for an apparent scaling behavior of

$$
\frac{\varepsilon_{\phi}^{2}}{\sigma_{\phi}^{2}}=K \cdot f_{3 d B}^{-\gamma}
$$

where $\varepsilon_{\phi}^{2}$ is the residual phase variance with $\mathrm{AO}$ compensation, $\sigma_{\phi}^{2}$ is the open-loop phase variance, $\gamma$ is an power dependent upon the properties of the random disturbance and the assumed control law, and $K$ is an arbitrary constant of proportionality. Fig. 3 shows plots of $\ln \left(\varepsilon_{\phi}^{2} / \sigma_{\phi}^{2}\right)$ as a function of $-\ln \left(f_{3 d B}\right)$ for the compensation simulation with $130^{\circ}$ turret angle. The residual phase variance was computed given the measured higher-order Strehl ratio $S_{h}$ and applying the Maréchal approximation, ${ }^{13} \varepsilon_{\phi}^{2} \simeq-\ln \left(S_{h}\right)$. As expected a strong linear relation is observed for the unfiltered and filtered data (low, high frequencies). From the slope and intercept of a linear fit to the simulation data, the values of $\gamma$ and $K$ in Eq. (6) may be determined. Substituting $K=f_{A}^{\gamma} / 2$, it follows that

$$
\varepsilon_{\phi}^{2}=\frac{1}{2} \sigma_{\phi}^{2}\left(\frac{f_{A}}{f_{3 d B}}\right)^{\gamma},
$$

where $f_{A}$ may be interpreted as a compensation scaling frequency for the aero-optical disturbance. By definition, when $f_{3 d B}=f_{A}$, the aero-optics phase variance is reduced by a factor of 2 . In a consistent manner, a scaling law for the compensated aero-optics higher-order Strehl ratio may be computed as

$$
S_{h} \simeq \exp \left[-\frac{1}{2} \sigma_{\phi}^{2}\left(\frac{f_{A}}{f_{3 d B}}\right)^{\gamma}\right]
$$

Table 1 gives a summary of the compensation scaling law parameters derived from the AO simulations for the scaled wind-tunnel aero-optics disturbances. For $130^{\circ}$ turret angle with the unfiltered data, $f_{A}=71$ $\mathrm{Hz}$ indicates that a moderate-bandwidth $\mathrm{AO}$ compensation loop supported by current $\mathrm{AO}$ technology could yield significant compensation of aero-optical disturbances. The majority of the Strehl improvement with AO 


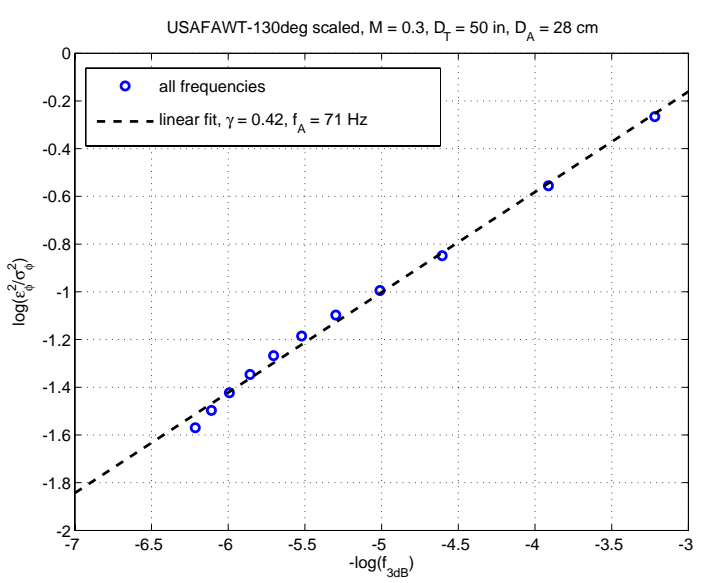

(a) Unfiltered aero-optics data

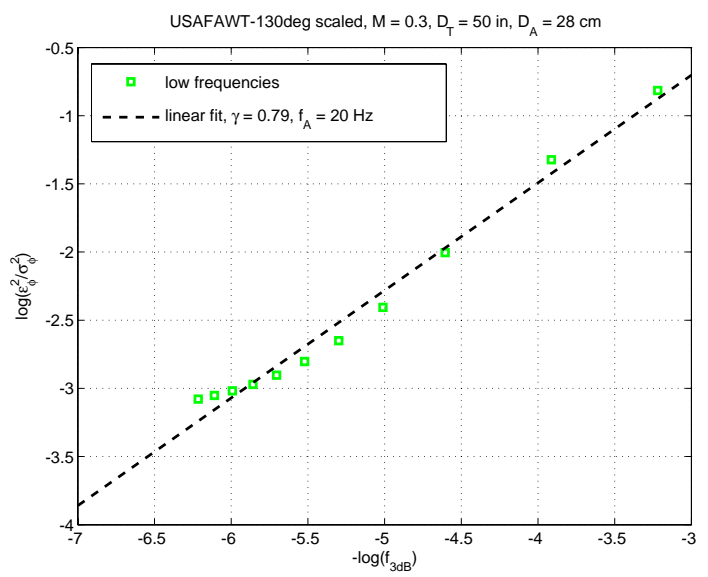

(c) Low-frequency aero-optics data $(f<144 \mathrm{~Hz})$

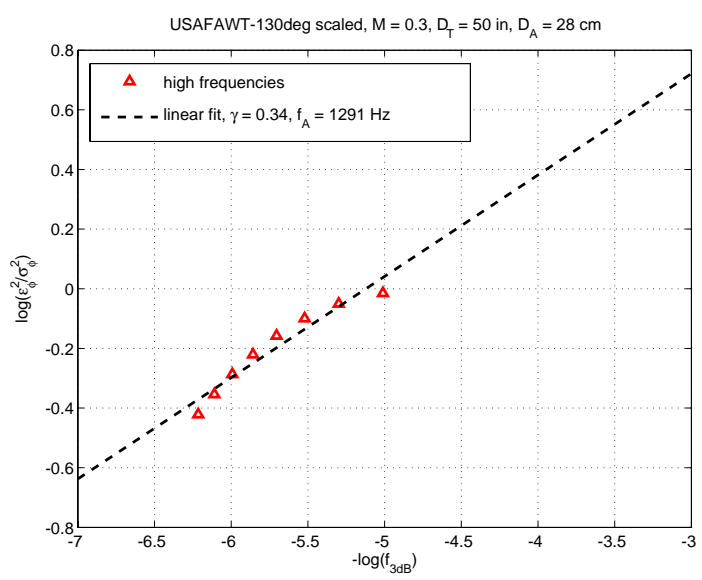

(e) High-frequency (shear layer) aero-optics data $(f>144 \mathrm{~Hz})$

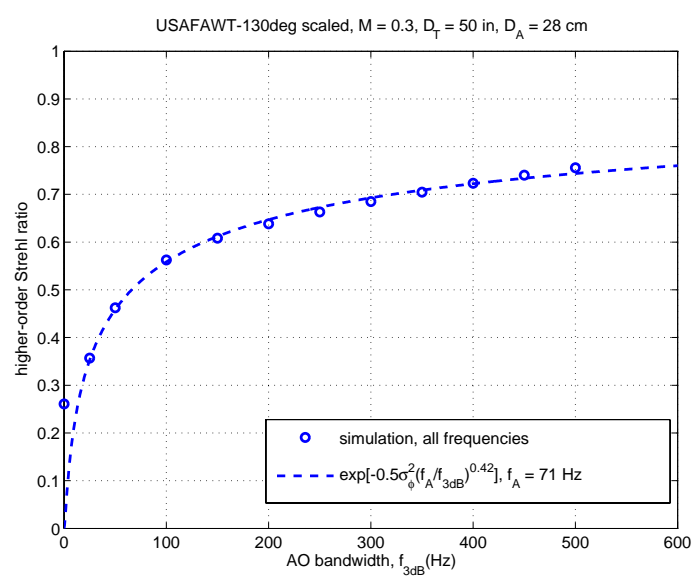

(b) Compensation scaling law, unfiltered

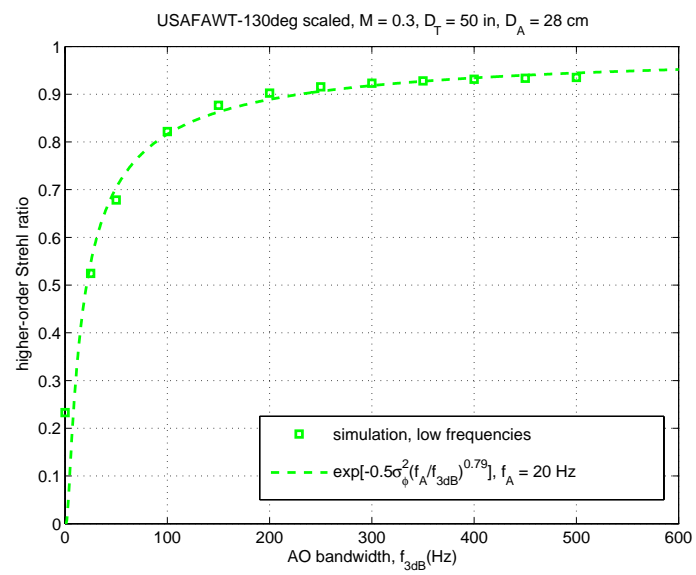

(d) Compensation scaling law, low frequency

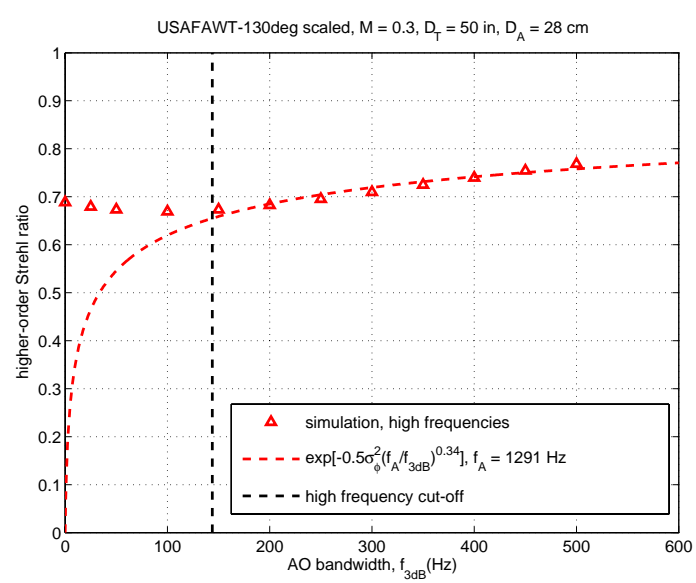

(f) Compensation scaling law, high frequency

Figure 3. Analysis of AO compensation performance with classical control (zero latency) for $130^{\circ}$ turret angle. Plots show the compensation scaling law, as fit to the simulation data. 
compensation comes from correcting low-frequency disturbances well, while the high-frequency aero-optical disturbances are essentially uncorrected. The compensation frequency for the high-frequency disturbances is nearly 2 orders of magnitude higher than for the low-frequency disturbances and the power associated with compensating these aberrations is $\gamma \sim 1 / 3$. The high frequency shear-layer disturbance is a smaller contributor to optical degradation than the low-frequency disturbance.

Table 1. Summary of scaling law parameters determined from AO compensation simulations.

\begin{tabular}{|c|c|c|c|c|}
\hline turret angle & disturbance & $\sigma_{\phi}(\lambda)$ & $\gamma$ & $f_{A}(\mathrm{~Hz})$ \\
\hline \hline $130^{\circ}$ & unfiltered & 0.19 & 0.42 & 71 \\
\hline $130^{\circ}$ & low frequency & 0.19 & 0.79 & 20 \\
\hline $130^{\circ}$ & high frequency & 0.10 & 0.34 & 1291 \\
\hline
\end{tabular}

\section{III.B. Effect of latency on compensation performance}

In practice integration and readout from wavefront sensors, wavefront reconstruction, control processing, and response from correction devices do not happen instantaneously but instead require a finite amount of time to complete. A well-designed AO system can typically accomplish all these steps by the time the next WFS frame begins integration. However, it is not uncommon for the net latency to be greater than 1 frame since WFS integration and readout alone may take up a good portion of the sensor framing interval. The net system latency will in general degrade system performance. The nature and magnitude of performance degradation depends on the temporal properties of the disturbance and the error rejection properties of such a control loop. For classical control with "bandwidth" $f_{B W}$ and latency $\Delta t$, the error rejection for the controller is modeled theoretically as

$$
E R J(f)=\left[1+\left(\frac{f_{B W}}{f}\right)^{2}-2\left(\frac{f_{B W}}{f}\right) \sin (2 \pi f \Delta t)\right]^{-1} .
$$

For the limiting case of $\Delta t=0$, then Eq. (9) and Eq. (4) are equivalent, and $f_{B W}$ has the same connotation as $f_{3 d B}$.

Fig. 4 shows a plot of the measured error rejection from our simulation using $\beta=0.18\left(f_{B W}=100 \mathrm{~Hz}\right)$ and $\beta=0.36\left(f_{B W}=200 \mathrm{~Hz}\right)$. The simulation data for both bandwidths was fit to the theoretical model yielding an optimal value of $\Delta t=440 \mu \mathrm{sec}$. Fig. 4 illustrates that the error rejection characteristic of the classical AO controller is well modeled by the applicable theory. Note that for $f_{d}>150 \mathrm{~Hz}$, the classical controller exhibits significant amplification of the input disturbance.

Given the error rejection function for the classical AO controller in Eq. (9) its ability to model the error rejection properties of our $\mathrm{AO}$ simulation, the residual phase variance for a particular disturbance can be computed as

$$
\varepsilon_{r e s}^{2}=\int_{0}^{\infty} E R J\left(f ; f_{B W}, \Delta t\right) \Phi_{d}(f) d f
$$

where $\Phi_{d}(f)$ is the PSD of the disturbance to be compensated. The predicted compensated Strehl ratio for classical AO can be computed using Eq. (10) and approximating $S_{h, \text { aero }} \simeq \exp \left(-\varepsilon_{\text {res }}^{2}\right)$. If we consider specifying a frequency $f_{S L}$ above which are associated the shear-layer disturbances, then we may rewrite Eq. (10) without loss of generality as

$$
\begin{aligned}
\varepsilon_{\text {res }}^{2} & =\int_{0}^{f_{S L}} E R J\left(f ; f_{B W}, \Delta t\right) \Phi_{d}(f) d f+\int_{f_{S L}}^{\infty} E R J\left(f ; f_{B W}, \Delta t\right) \Phi_{d}(f) d f, \\
& =\varepsilon_{\text {res: low }}^{2}+\varepsilon_{\text {res: high }}^{2},
\end{aligned}
$$

from which it follows that

$$
\begin{aligned}
& S_{h, \text { aero }}=\exp \left(-\varepsilon_{\text {res: }}^{2} \text { low }\right) \exp \left(-\varepsilon_{\text {res: high }}^{2}\right), \\
& S_{h, \text { aero }}=S_{h, \text { aero: } \text { low }} \cdot S_{h, \text { aero: } \text { high }} .
\end{aligned}
$$

Hence we would anticipate that the composite aero-optical compensation characteristic would be a product of the low-frequency and high-frequency behavior. 


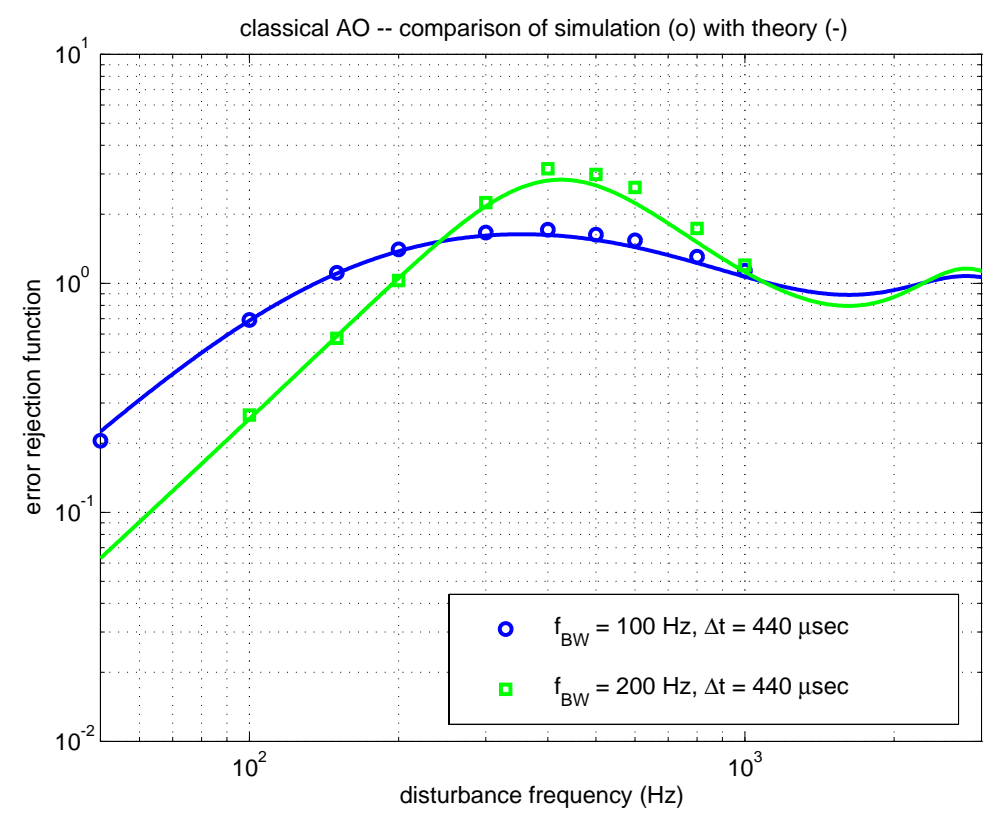

Figure 4. Measured error rejection characteristic for classical AO control with gain set to $\beta=0.18\left(f_{B W}=100 \mathbf{H z}\right)$ and $\beta=0.36\left(f_{B W}=200 \mathbf{H z}\right)$ compared to the theoretical model. The optimal fit of the measurement to the theoretical model was given by $\Delta t=440 \mu$ sec.

optimum between $100 \mathrm{~Hz}$ and $150 \mathrm{~Hz}$. For low-frequency disturbances, there is essentially no differen in compensation performance with increased latency regardless of AO bandwidth. For the high-frequency shear-layer disturbances, however, the AO latency leads to severe degradation with increasing bandwidth. Note that for $\Delta t=440 \mu \mathrm{sec}$, the compensated Strehl ratio is always lower than the open-loop, indicating an overall amplification of the disturbance by the controller. In this light, the optimization of the compensated Strehl for the full disturbance can be understood as the benefit of strong attenuation of the large-magnitude, low-bandwidth disturbances being offset by amplification of the high-bandwidth shear-layer. It is this amplification of disturbances at high-frequency that the adaptive controller can overcome.

\section{Adaptive control of aero-optics and atmospheric disturbances}

\section{IV.A. Adaptive control method for adaptive optics}

Fig. 6 shows a block diagram of the AO controller used for simulations in this study. For design of the control loops, it is assumed that WFS vector $y$ and DM command vector $c$ are related by

$$
y=w-\Gamma c,
$$

were $\Gamma$ is the poke matrix and $w$ is the part of the WFS vector due to the wavefront error produced by atmospheric turbulence. The top two feedback loops in the block diagram in Figure 6 are classical AO and track loops, with integrator gains $k_{1}$ and $k_{2}$, respectively. The matrix $V$ in the AO loop defines a parametrization of actuator space. The columns of $V$ represent DM modes that are commanded independently by the control loops. Thus,

$$
c=V v
$$

where the vector $v$ contains the independent control commands generated by the AO loops. This study used a set of frequency-weighted DM modes computed according to the method introduced in a recent paper. ${ }^{9}$ Also, wavefront tilt is removed from the DM modes used here, so that the AO and track loops are uncoupled - at least to the extent that the linear model in Eq. (13) is valid. Such parameterizations have been used in, ${ }^{8,14,15}$ but the new method for computing frequency weighted DM modes produces more desirable DM modes. ${ }^{9}$ The reconstructor matrix $E_{0}$ in the classical AO loop is chosen to satisfy

$$
E_{0} \Gamma V=I \text {. }
$$




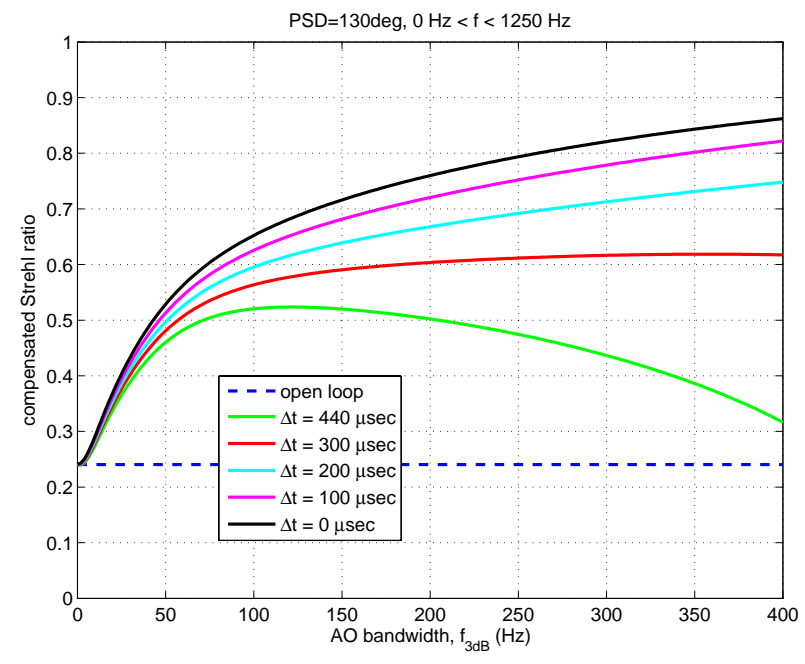

(a) Unfiltered aero-optics data

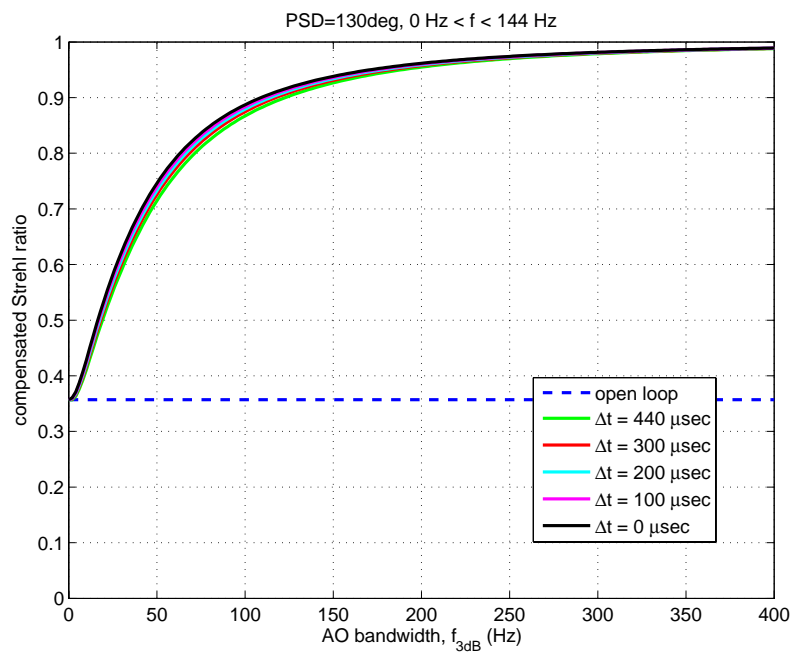

(b) Low-frequency aero-optics data $(f<144 \mathrm{~Hz})$

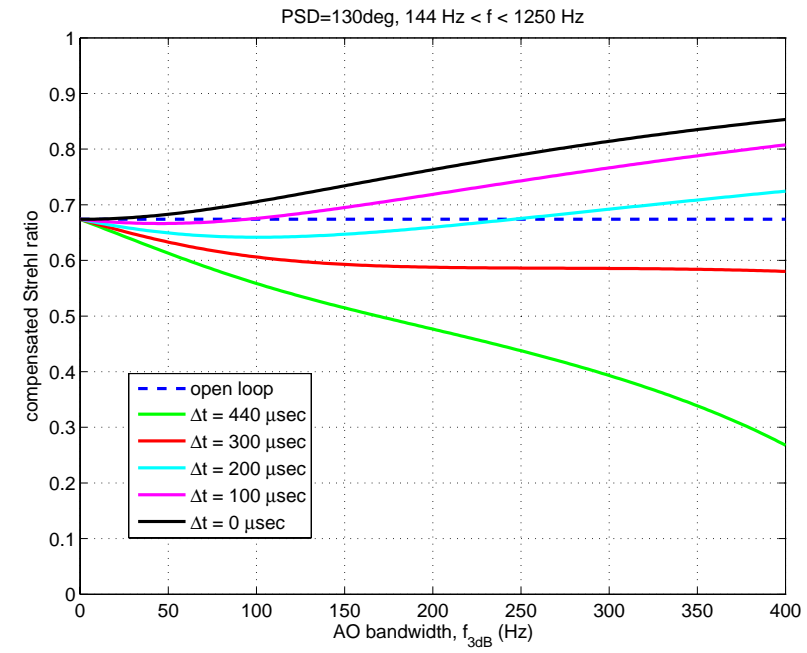

(c) High-frequency (shear layer) aero-optics data $(f>144 \mathrm{~Hz})$

Figure 5. Aero-optics compensation model for conventional AO given disturbance power spectrum for $130^{\circ}$ turret angle. AO latency degrades performance of AO compensation with higher loop gain (greater bandwidth, $f_{3 d B}$ ). 
The adaptive control loop is enclosed in the dashed box in the block diagram in Figure 6. This control loop augments the classical AO loop to enhance wavefront prediction and correction, particularly for higher-order wavefront modes. The main component of this loop is the lattice filter $L(z)$. The form of $L(z)$ used here is based on the multichannel lattice filter developed at UCLA, ${ }^{16}$ which derives numerical stability and efficiency from an orthogonalization of the data channels.

For adaptive identification of the filter gains, the problem is formulated as a feed-forward disturbance-rejection problem with reference signal

$$
r=G(z) u-E_{1} y
$$

and tuning signal

$$
e=E_{1} y
$$

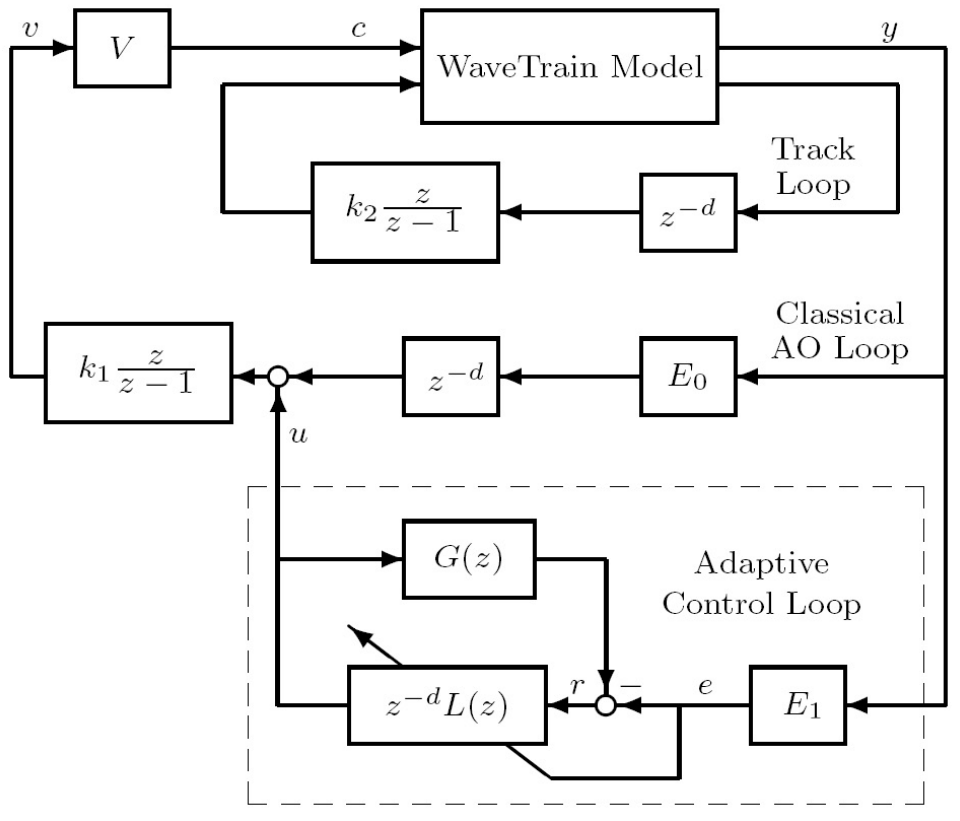

Figure 6. Control loops closed around the WaveTrain simulation of the laser compensation system, including the classical AO loop and the adaptive augmentation.

as indicated in Figure 6. The transfer function $G(z)$ is an approximation to the transfer function from the control signal $u$ to the tuning signal $e$ with only the classical AO loop closed, and $E_{1}$ is a constant matrix. Thus, the input to the filter $L(z)$ is $r$, and the gains in $L(z)$ are identified to minimize the RMS value over time and space of $e$ (equivalently, the RMS value over time of $\|e\|$ ).

With the hypothesis in Eq. (13), the condition Eq. (15) and $E_{1}=E_{0}$, the control channels represented by the columns of the matrix $V$ are uncoupled in the classical AO loop. The transfer function $G(z)$ then reduces to the following scalar transfer function for each channel:

$$
G(z)=\frac{-k_{1} z^{d}}{z^{d}-z^{d-1}+k_{1}} .
$$

The modeling error between this transfer function and the true transfer function from $u$ to $e$ results from the error between Eq. (13) and the true mapping from the DM commands to the WFS vector. Extensive simulation results have demonstrated that the adaptive loop compensates for this modeling error.

\section{IV.B. Compensation of aero-optics}

We first consider simulation of adaptive control for compensation of aero-optics only. Fig. 7 shows the performance of classical and adaptive AO for control bandwidths in the range $0 \leq f_{3 d B} \leq 400$. The simulation runs were made with unfiltered $130^{\circ}$ aero-optics data. Fig. $7($ a) shows two plots for adaptive control; one for the adaptive loop augmentation with a constant gain $k_{1}=0.5$ and one with $k_{1}=\beta$, where $\beta$ is the loop gain for the classical controller for the specified bandwidth; $f_{3 d B}=\beta f_{s} /(2 \pi)$-see Eq. $(5)$. These alternatives for adaptive augmentation gain were chosen based on loop stability intervals addressed in previous evaluations of the adaptive controller. ${ }^{9}$ In general, $k_{1}=\beta$ has better stability properties for augmentation of low-bandwidth classical AO, whereas $k_{1}=0.5$ has better properties when coupled with high-bandwidth control. The performance of either option for adaptive control yields similar performance improvement in the AO loop, giving $1.5 \times$ to $2.0 \times$ improvement in the compensated Strehl ratio relative to classical AO control.

Fig. 8 shows simulation results classical AO and adaptive control for the high-frequency aero-optics (shearlayer) disturbances only. The classical AO simulations in Fig. 8(a) exhibit the behavior expected given the analysis of Sec. III.B-see Fig. 5. With $440 \mu \mathrm{sec}$ of AO latency, classical control always degrades the Strehl ratio and continues to amplify the high-frequency disturbance as the bandwidth increases. Adaptive control rectifies this degradation, offering a compensated Strehl ratio $S_{h} \geq 0.75$ over the interval studied. Overall, 


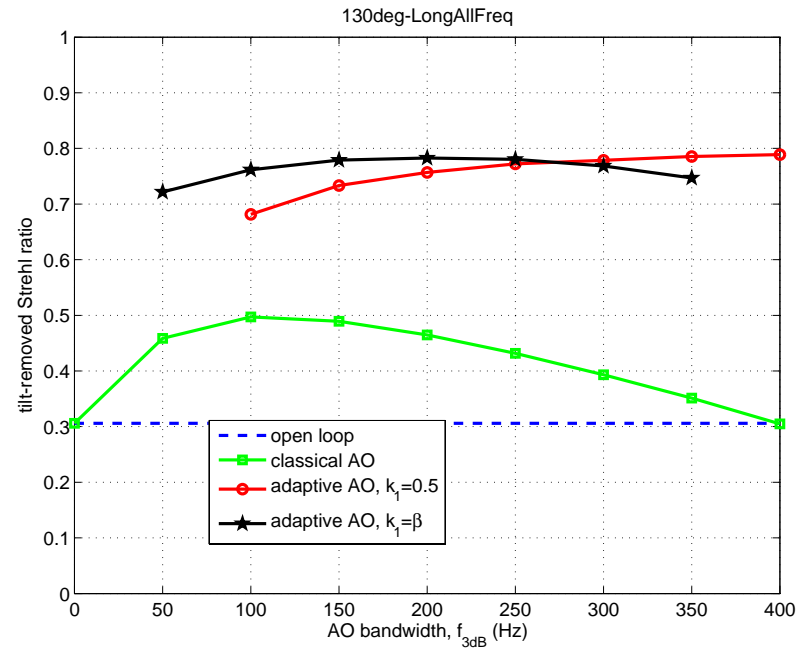

(a) Compensated Strehl ratio with classical AO, adaptive AO $\left(k_{1}=0.5,\right)$ and adaptive $\mathrm{AO}\left(k_{1}=\beta\right.$. $)$

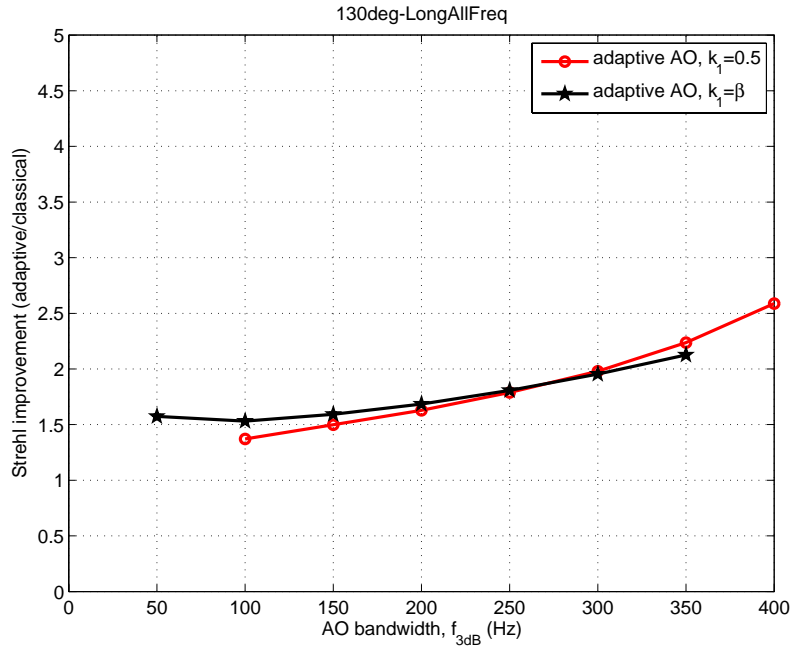

(b) Performance improvement with adaptive control relative to classical AO.

Figure 7. AO performance enhancement with adaptive control for aero-optical disturbances only, $130^{\circ}$ turret angle, all frequencies (unfiltered.)

the adaptive control gives $1.25 \times$ to $1.5 \times$ increase in the compensated Strehl. It should be noted that the adaptive control Strehl results shown in Fig. 8 for $50 \mathrm{~Hz}$ and $100 \mathrm{~Hz} \mathrm{AO}$ bandwidth with $440 \mu$ sec latency are better than those achieved at $500 \mathrm{~Hz}$ bandwidth and zero latency in the idealized simulations of Sec. III.Asee Fig. 3(c). This is an important result to recognize, as it clearly demonstrates that adaptive control can effectively remove the degradation owing to AO latency while offering compensation performance equivalent to a $10 \times$ increase in error rejection bandwidth with classical AO control.

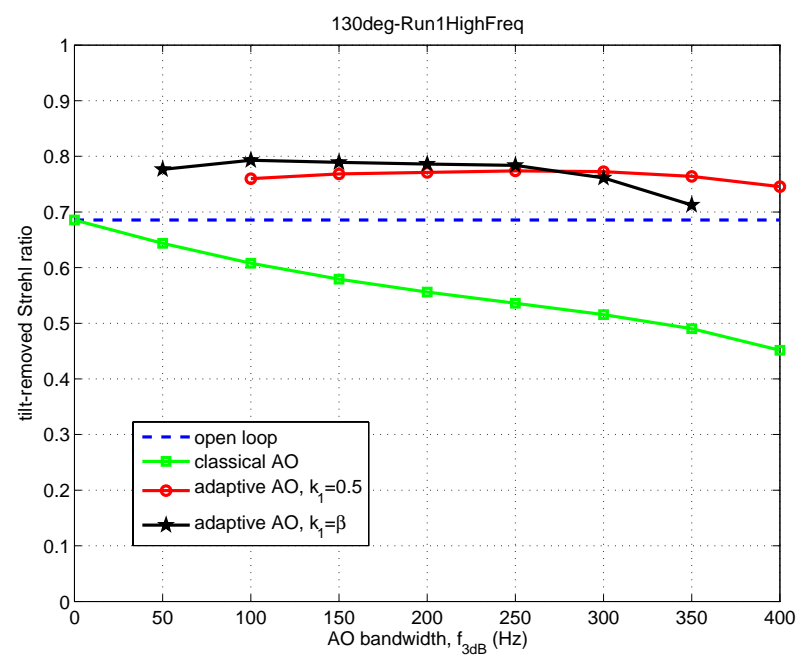

(a) Compensated Strehl ratio with classical AO, adaptive AO $\left(k_{1}=0.5,\right)$ and adaptive $\mathrm{AO}\left(k_{1}=\beta.\right)$

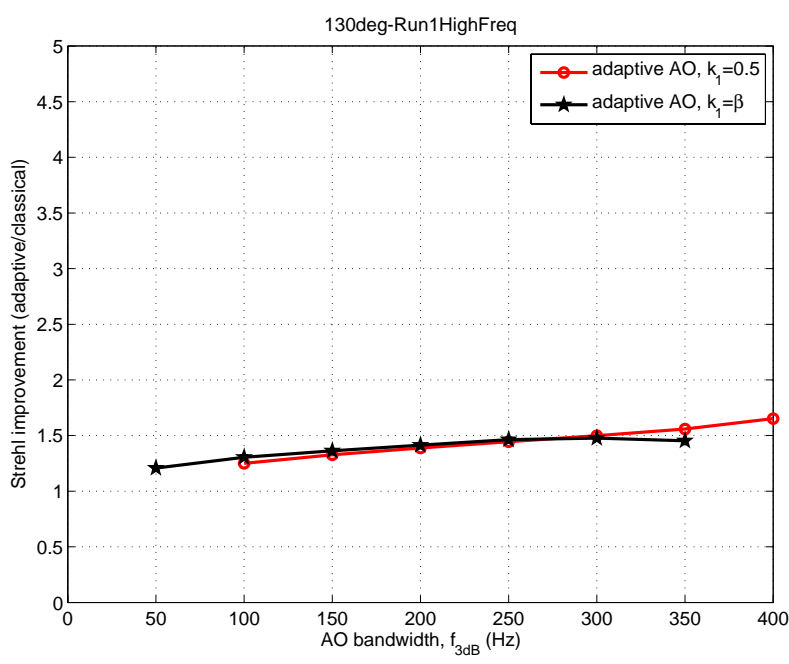

(b) Performance improvement with adaptive control relative to classical $\mathrm{AO}$

Figure 8. AO performance enhancement with adaptive control for aero-optical disturbances only, $130^{\circ}$ turret angle, high-frequency shear-layer.

\section{IV.C. Compensation of free-stream turbulence}

The adaptive controller also has the ability to improve compensation performance for free-stream turbulence. Fig. 9 shows simulations results of classical and adaptive AO control for the case of free-stream turbulence only with $D / r_{0}=3, f_{G}=243 \mathrm{~Hz}$. Under these propagation conditions, it is anticipated that $f_{3 \mathrm{~dB}}>f_{G}$ 
would be required to achieve adequate turbulence compensation. However, the latency $\Delta t=440 \mu$ sec will limit high-bandwidth compensation performance. From Fig. 9(a) we see that compensation performance with classical $\mathrm{AO}$ control is optimized for $f_{3 d B} \simeq 300 \mathrm{~Hz}$. Adaptive control offers significant performance enhancement over the full interval of $\mathrm{AO}$ bandwidths simulated. The $k_{1}=0.5$ and the $k_{1}=\beta$ options for the adaptive controller give nearly equal performance. The Strehl ratio enhancement is nearly constant with $\mathrm{AO}$ bandwidth. The adaptive controller gives $\sim 1.5 \times$ increase in Strehl for the $D / r_{0}=3, f_{G}=243 \mathrm{~Hz}$ free-stream propagation conditions.

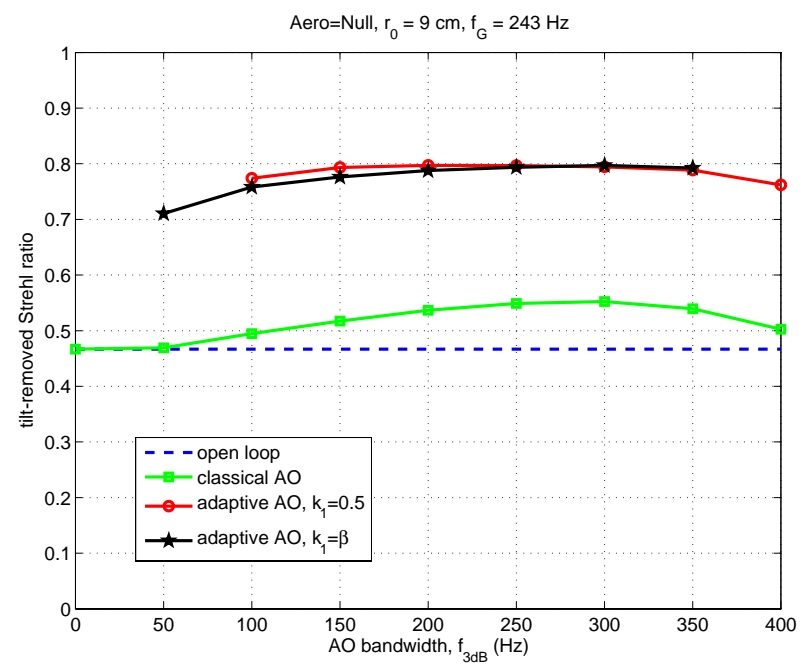

(a) Compensated Strehl ratio with classical AO, adaptive AO $\left(k_{1}=0.5,\right)$ and adaptive $\mathrm{AO}\left(k_{1}=\beta\right.$. $)$

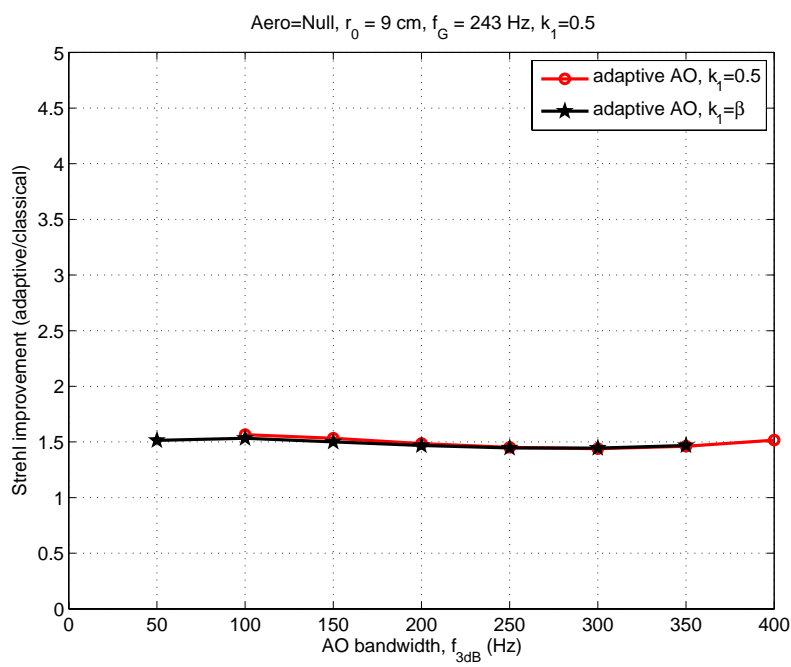

(b) Performance improvement with adaptive control relative to classical AO.

Figure 9. AO performance enhancement with adaptive control for free-stream disturbances only, $D / r_{0}=3, f_{G}=243 \mathrm{~Hz}$.

\section{IV.D. Combined aero-optics and free-stream turbulence compensation}

Simulation for aero-optics only in Sec. IV.B and free-stream turbulence only in Sec. IV.C showed that adaptive control offered a substantial performance enhancement over the baseline classical AO. Since a practical aircraft laser compensation system must deal with both aero-optics and free-stream turbulence, then it is important to simulate performance when both these disturbances are in the laser path.

Fig. 10 shows the comparison of classical and adaptive $\mathrm{AO}$ for $130^{\circ}$ turret angle aero-optics with $\mathrm{D} / \mathrm{r}_{0}=3$ and $f_{G}=243 \mathrm{~Hz}$ in the free-stream. Fig. 10(b) shows that with adaptive control, the compensated Strehl ratio can be increased by a factor of $2.5 \times$ to greater than $3.5 \times$, depending on the AO bandwidth. The same trend for setting the adaptive loop gain is noted $-k_{1}=\beta$ is best for $\beta<0.5$, whereas $k_{1}=0.5$ is gives superior performance for cases where $\beta>0.5$.

Table 2 summarizes our AO simulations results for classical and adaptive AO control for various freestream and aero-optical conditions with $f_{3 d B}=200 \mathrm{~Hz}$ and $440 \mu \mathrm{sec}$ latency. The adaptive control results quoted in Table 2 are for $k_{1}=\beta$, which generally gave better performance at $f_{3 d B}=200 \mathrm{~Hz}$ than $k_{1}=0.5$. The $D / r_{0}=0$ and $f_{G}=0 \mathrm{~Hz}$ results refer to the simulations conducted with aero-optics only (no free-stream turbulence). The designator "NULL" for the aero-optics sequence indicates that no aero-optical disturbances were included in the simulation.

The trend that we note from these results is that as the composite propagation conditions become more severe, the adaptive controller provides more benefit to AO compensation. This trend is due in part to the fact that the classical controller performance degrades while the adaptive AO controller is relatively robust to varying propagation conditions. A close examination of the performance improvement factors for aero-optics and free-stream turbulence tested separately compared to the performance improvement with both effects present in the path will show that the performance improvement is nearly multiplicative. For example, with aero-optics only, the Strehl enhancement was 1.68. With free-stream turbulence only and $D / r_{0}=3, f_{G}=243 \mathrm{~Hz}$, the Strehl enhancement is 1.47. With both effects present, the enhancement is $2.44 \simeq 1.68 \times 1.47=2.47$. Fig. 11 shows the progression of compensated laser cross-sections for these cases. 


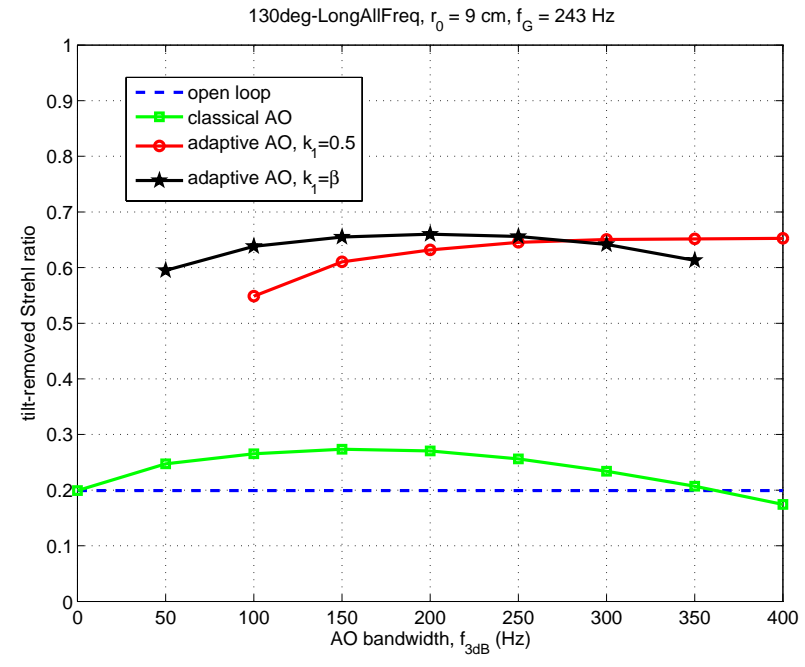

(a) Compensated Strehl ratio with classical AO, adaptive AO $\left(k_{1}=0.5,\right)$ and adaptive $\mathrm{AO}\left(k_{1}=\beta\right.$. $)$

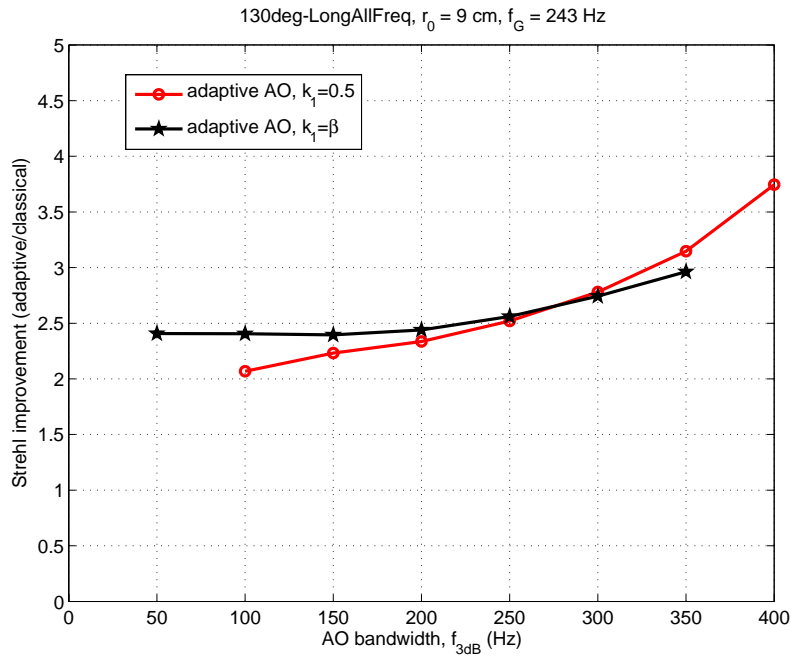

(b) Performance improvement with adaptive control relative to classical AO.

Figure 10. AO performance enhancement with adaptive control for combined aero-optics ( $130^{\circ}$ turret angle) and free-stream turbulence $\left(D / r_{0}=3, f_{G}=243 \mathbf{H z}.\right)$

Table 2. Higher-order Strehl ratio improvement factors achieved with adaptive controller (gain $k_{1}=\beta=0.36$ ) compared to classical AO with $f_{3 d B}=200 \mathrm{~Hz}$ and $440 \mu$ sec latency.

\begin{tabular}{|c|c|c|c|c|c|}
\hline$D / r_{0}$ & $f_{G}(\mathrm{~Hz})$ & aero sequence & $S_{h}$, classical AO & $S_{h}$, adaptive AO & Strehl improvement \\
\hline \hline 0 & 0 & $130^{\circ}$ Long & 0.46 & 0.78 & $\mathbf{1 . 6 8}$ \\
\hline \hline 2 & 158 & NULL & 0.71 & 0.84 & $\mathbf{1 . 1 7}$ \\
\hline 2 & 158 & $130^{\circ}$ Long & 0.35 & 0.70 & $\mathbf{2 . 0 2}$ \\
\hline 2 & 296 & $130^{\circ}$ Long & 0.33 & 0.68 & $\mathbf{2 . 0 6}$ \\
\hline \hline 3 & 243 & NULL & 0.54 & 0.79 & $\mathbf{1 . 4 7}$ \\
\hline 3 & 243 & $130^{\circ}$ Long & 0.27 & 0.66 & $\mathbf{2 . 4 4}$ \\
\hline 3 & 454 & $130^{\circ}$ Long & 0.25 & 0.64 & $\mathbf{2 . 6 0}$ \\
\hline
\end{tabular}

\section{Conclusion}

Performance of a classical AO compensation loop was quantified using wind tunnel measurements of aero-optical disturbances. AO performance was characterized in terms of a higher-order Strehl ratio $S_{h}$ as a function of the error-rejection bandwidth $f_{3 d B}$ of the $\mathrm{AO}$ control loop. It was shown that the residual phase variance with $\mathrm{AO}$ control, when normalized to the open-loop, scales as $\left(f_{A} / f_{3 d B}\right)^{\gamma}$, where $\gamma$ is an arbitrary power and $f_{A}$ is a characteristic frequency of the aero-optical disturbance determined from a linear fit of the compensation data with increasing bandwidth. The frequency $f_{A}$ is defined as the bandwidth for which the residual phase variance is one-half of the open-loop value. For $130^{\circ}$ turret angle, it was shown that $\gamma=0.42$ and $f_{A}=71 \mathrm{~Hz}$. The disturbance is shown to be dominated by low-frequency wavefront components for which $\gamma=0.79$ and $f_{A}=20 \mathrm{~Hz}$. High-frequency shear layer components were observed to be less than one-half as large (in an rms sense) as the low-frequency disturbances and also exhibit scaling behavior with $\gamma=0.34$ and $f_{A}=1291 \mathrm{~Hz}$. These studies indicated that an AO system with $200 \mathrm{~Hz}$ error rejection bandwidth may substantively compensate the low-frequency aero-optical aberrations, a major contributor to the optical degradation. However, the high characteristic frequencies and low scaling powers for the shear layer disturbances are a limiter to the performance of classical AO operating at moderate error rejection bandwidth. The deleterious effects of aero-optics shear layer disturbances are more pronounced at higher platform velocities and will be exacerbated if the AO system suffers from latency.

To address the observed limitations for classical AO control, we have developed an adaptive control 


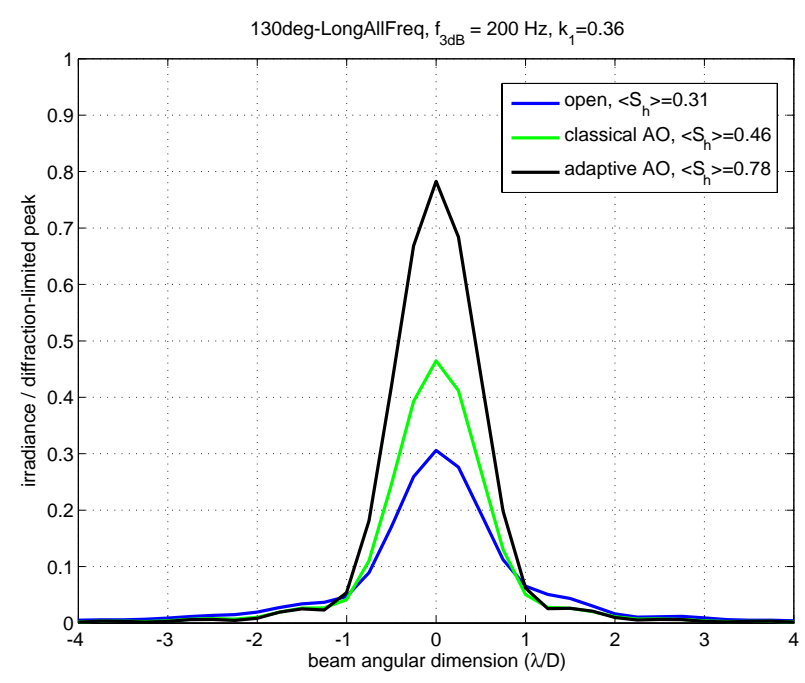

(a) Aero-optics only, $130^{\circ}$ turret angle.

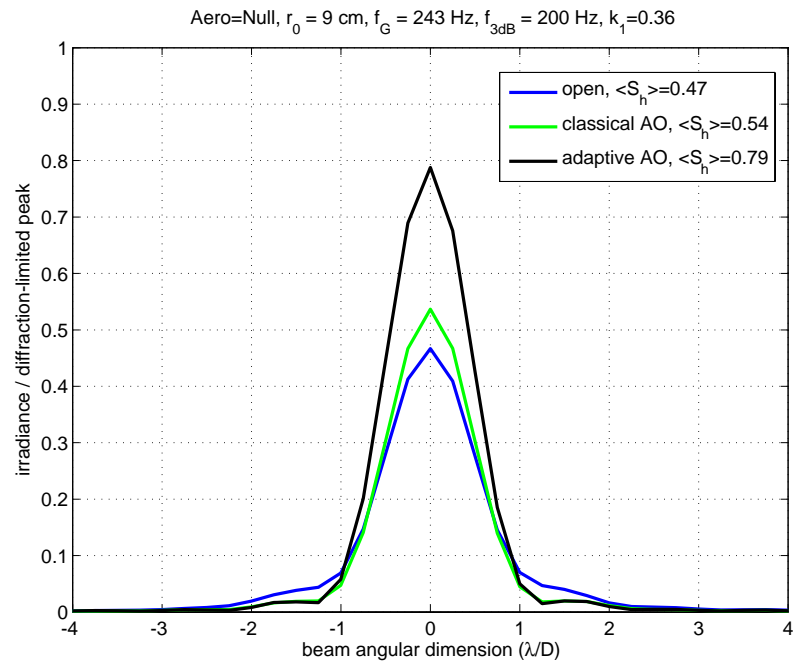

(b) Free-stream turbulence only, $D / r_{0}=3, f_{G}=243 \mathrm{~Hz}$.

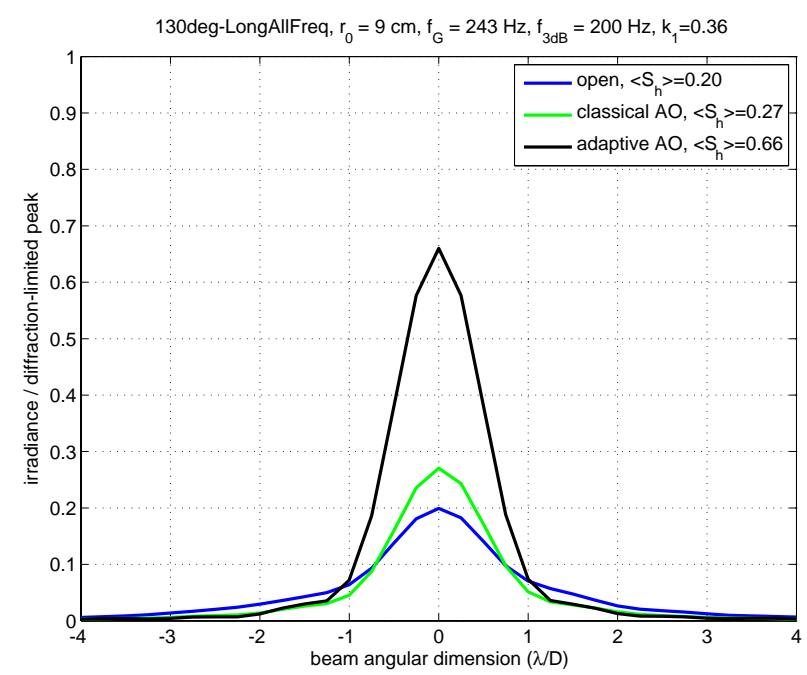

(c) Combined aero-optics and free-stream turbulence, $130^{\circ}$ turret angle, $D / r_{0}=3, f_{G}=243 \mathrm{~Hz}$.

Figure 11. Progression of adaptive AO control compensated laser cross-sections.

algorithm that may be used with the same sensor and compensation hardware as required for classical control. The adaptive control for aero-optics builds on previous work applying this method to adaptive optics for compensating free-stream turbulence. The same formalism and basic design was applied to the current problem of aero-optics compensation, including the fundamental lattice filtering approach for the adaptive loop augmentation.

Extensive wave-optics simulations were conducted using the WaveTrain ${ }^{\mathrm{TM}}$ model with classical AO and adaptive control. Compensation of aero-optics disturbances and free-stream turbulence were conducted separately, and then later combined in the simulation. Simulation with variable classical AO loop gain showed performance trends consistent with theoretical analysis. When adaptive control was applied to aero-optical disturbances only, the compensated Strehl ratio increased by a factor of $1.5 \times$ to $2.0 \times$. When shear-layer disturbances were considered, it was found that adaptive control can effectively remove the degradation owing to $\mathrm{AO}$ latency while offering compensation performance equivalent to a $10 \times$ increase in error rejection bandwidth with classical AO control. Thus, a $50 \mathrm{~Hz}$ adaptive control loop with $440 \mu$ sec latency could perform as well as a $500 \mathrm{~Hz}$ classical AO loop with zero latency. The performance enhancement for adaptive control of free-stream turbulence varied with $D / r_{0}$ and the Greenwood frequency, with more enhancement noted for more severe turbulence conditions. Using combined aero-optics and free-stream turbulence, compensated 
Strehl ratio could be improved by a factor of $2.0 \times$ to $4.0 \times(2.5 \times$ typical $)$, depending upon the nature of the free-stream turbulence and the bandwidth of the classical AO baseline. The enhancement for combined effects was nearly equal to the product of enhancement when each effect was tested separately. In comparison with classical AO, compensation performance with adaptive control was robust to changes in the state and severity of disturbance conditions.

\section{Acknowledgment}

This work was performed under Small Business Innovative Research (SBIR) contract number FA945106-M-0128 sponsored by the Air Force Research Laboratory (AFRL). This paper was cleared for public dissemination by the Directed Energy Directorate Public Affairs Office (AFRL/DEO-PA) under case \#07260, 31 May 2007.

\section{References}

\footnotetext{
${ }^{1}$ Gordeyev, S., Post, M. L., McLaughlin, T., Ceniceros, J., and Jumper, E. J., "Survey of Optical Environment over Hemisphere-on-Cylinder Turret Using Suite of Wavefront Sensors," 37th AIAA Plasmadynamics and Lasers Conference, Vol. 3074, 2006.

${ }^{2}$ Greenwood, D. P., "Bandwidth specifications for adaptive optics systems," J. Opt. Soc. Am., Vol. 67, No. 3, March 1977, pp. 390-393.

${ }^{3}$ Gibert, K. G. and Otten, L. J., Aero-Optical Phenomena, American Institute of Aeronautics and Astronautics, New York, 1982.

${ }^{4}$ Fitzgerald, E. J. and Jumper, E. J., "The Optical Distortion Mechanism in a Nearly Incompressible Free Shear Layer," Journal of Fluid Mechanics, Vol. 512, 2004, pp. 153-189.

${ }^{5}$ Gordeyev, S., Hayden, T., and Jumper, E. J., "Aero-Optical and Flow Measurements over a Flat-Windowed Turret," AIA A Journal, Vol. 45, No. 2, 2007, pp. 347-357.

${ }^{6}$ Tyler, G. A. et al., "Adaptive optics: Theory and applications," Tech. Rep. AFRL-DE-PS-TR-1998-1054, Air Force Research Laboratory, Directed Energy Directorate, Kirtland AFB, New Mexico, 1999, Contract Number F29601-94-C-0133, the Optical Sciences Company (tOSC).

${ }^{7}$ Roggemann, M. C. and Welsh, B., Imaging Through Turbulence, CRC Press, Boca Raton, 1996.

${ }^{8}$ J. S. Gibson, C.-C. Chang, and B. L. Ellerbroek, "Adaptive Optics: wavefront correction by Use of Adaptive Filtering and Control," Applied Optics, Optical Technology and Biomedical Optics, Vol. 39, No. 16, June 2000, pp. 2525-2538.

${ }^{9}$ Liu, Y.-T. and Gibson, J. S., "Adaptive Control in Adaptive Optics for Directed Energy Systems," Optical Engineering, Vol. 46, No. 4, 2007, pp. 046601-1-046601-13.

${ }^{10}$ MZA Associates Corporation, "WaveTrain: Wave optics made easier," On the world-wide web at http://www.mza.com.

${ }^{11}$ Ellerbroek, B. L., "First-order performance evaluation of adaptive-optics systems for atmospheric turbulence compensation in extended-field-of-view astronomical telescopes," J. Opt. Soc. Am. A, Vol. 11, No. 2, February 1994, pp. 783-805.

${ }^{12}$ Tyler, G. A., "Bandwidth considerations for tracking through turbulence," J. Opt. Soc. Am. A, Vol. 11, No. 1, January 1994, pp. 358-367.

${ }^{13}$ Born, M. and Wolf, E., Principles of Optics, Pergamon Press, Oxford, sixth ed., 1980.

${ }^{14}$ C.-C. Chang and J. S. Gibson, "Parallel Control Loops Based on Spatial Subband Processing for Adaptive Optics," American Control Conference, IEEE, Chicago, June 2000.

${ }^{15}$ J. S. Gibson, C.-C. Chang, and Neil Chen, "Adaptive Optics with a New Modal Decomposition of Actuator and Sensor Spaces," American Control Conference, Arlington, VA, June 2001.

${ }^{16}$ S.-B. Jiang and J. S. Gibson, "An Unwindowed Multichannel Lattice Filter with Orthogonal Channels," IEEE Transactions on Signal Processing, Vol. 43, No. 12, December 1995, pp. 2831-2842.
} 